\title{
Tratamento minimamente invasivo da coledocolitíase e suas complicações: relato de caso
}

\author{
Case report: minimally invasive treatment of coledocholithiasis and its complications \\ Marina Miury Sato Rodrigues Alves ${ }^{1}$, Maíra Leite Basile ${ }^{1}$, Pedro Henrique Tsuyoshi Yamaoka ${ }^{1}$, \\ José Cesar Assef ${ }^{2}$, Tercio De Campos²
}

\begin{abstract}
Resumo
A Colangiopancreatografia Retrógrada Endoscópica (CPRE) é uma técnica de estudo das vias pancreatobiliares. Relatamos uma perfuração duodenal associada à hemorragia pós CPRE. Apesar de infrequentes, tais complicações possuem altas taxas de mortalidade, dependem sobretudo da experiência do endoscopista e seu tratamento de escolha é não cirúrgico.
\end{abstract}

Descritores: Pancreatocolangiografia Retrógrada Endoscópica, Duodeno/lesões, Coledocolitíase/complicações, Perfuração intestinal, Hemorragia

\begin{abstract}
Retrograde Endoscopic Cholangiopancreatography (ERCP) is a technique for the study of pancreatobiliary pathways. This is a case report on a duodenal perforation, post ERCP, associated with bleeding. Although infrequent, such complications have high mortality rates, they depend mainly on the experience of the endoscopist and their treatment of choice is non-surgical.
\end{abstract}

Keywords: Cholangiopancreatography, endoscopic retrograde; Duodenum/injuries; Choledocholithiasis/complications; Intestinal perforation; Hemorrhage

\section{Introdução}

Desde sua primeira descrição, em 1968, a Colangiopancreatografia Retrógrada Endoscópica (CPRE),

1. Acadêmico da Faculdade de Ciências Médicas da Santa Casa de São Paulo - $6^{\circ}$ ano do Curso de Graduação em Medicina

2. Professor Adjunto da Faculdade de Ciências Médicas da Santa Casa de São Paulo-Departamento de Cirurgia

Trabalho realizado: Irmandade da Santa Casa de Misericórdia de São Paulo - Departamento de Cirurgia

Endereço para correspondência: Marina Miury S. R. Alves. Rua Marquês de Itu, $n^{\circ} 382$, apto. 74 - Vila Buarque - 01223-000 - São Paulo-SP - Brasil técnica que associa a endoscopia e radiografias contrastadas para estudar e acessar os ductos da vesícula biliar, pâncreas e fígado, deixou de ter um papel somente diagnóstico e se tornou um procedimento preferencialmente terapêutico.

Suas complicações mais comuns são pancreatite, hemorragia e infecções, e podem ocorrer em 4 a 7\% dos $\operatorname{casos}^{(1)}$. Os principais fatores de risco associados são dificuldade na canulação do ducto biliar, cirrose, suspeita de disfunção do esfíncter de Oddi, técnica pré incisão e cirurgias gastrointestinais prévias.

O relato de caso a seguir diz respeito a duas dessas possíveis complicações, a perfuração duodenal e a hemorragia. A primeira é mais rara e corresponde a $0,37 \%$ delas ${ }^{(1)}$, sendo a retroperitoneal geralmente associada à esfincterotomia que se estende além da porção duodenal intramural (2). O sangramento, por sua vez, corresponde a $1,4 \%$, sendo na maioria dos casos de moderado a leve ${ }^{(1)}$.

\section{Relato de Caso}

Paciente MABN de 76 anos, mulher, branca, sem comorbidades e com antecedentes cirúrgicos de uma apendicectomia aos 25 anos de idade, dois partos cesáreas - o último há trinta e nove anos - e uma laqueadura há trinta e quatro, procurou o pronto-socorro do Hospital Central da Santa Casa de Misericórdia de São Paulo com quadro de dor abdominal intermitente e intensa há 15 dias, em região de epigástrio e hipocôndrio direito. Referia também episódios de acolia fecal e urina escurecida.

Ao exame, apresentava icterícia $3+/ 4+$, febre de $37,8^{\circ} \mathrm{C}$ e abdome doloroso à palpação profunda em hipocôndrio direito.

Aventadas as hipóteses diagnósticas de icterícia obstrutiva por provável colédocolitíase e colangite, foram solicitados exames laboratoriais e ultrassonografia de abdome. Obteve-se bilirrubina total de $11,0 \mathrm{mg} / \mathrm{dL}$ e direta de $9,0 \mathrm{mg} / \mathrm{dL}$, potássio de $5,4 \mathrm{mmol} / \mathrm{L}$, proteína $C$ reativa de $0,3 \mathrm{mg} / \mathrm{dL}$, gama glutil-transferase de 758 U/L e leucócitos de 12100/uL sem desvio à 
esquerda. O ultrassom evidenciou colelitíase com múltiplos cálculos móveis, o maior deles medindo $2,0 \mathrm{~cm}$, com dilatação de vias biliares intra e extra-hepáticas, associada ao aumento do calibre do colédoco $(0,9 \mathrm{~cm})$, sem identificação de fator obstrutivo.

A paciente foi internada e passou a receber ciprofloxacino e metronidazol.

No sexto dia de internação, apresentava-se estável e realizou CPRE, com laudo indicando papila de aspecto normal e difícil cateterização da via biliar, havendo necessidade de realizar papilotomia pela técnica de pré-corte com estilete, após a qual foi possível identificar anomalia de implantação do colédoco, que penetrava quase que perpendicular à parede do duodeno. O contraste injetado mostrou colédoco de tamanho normal com várias falhas de enchimento. Foi realizada dilatação da papila com balão dilatador e removidos microcálculos e areia biliar com balão extrator.

A paciente evoluiu com náuseas, distensão abdominal, taquicardia, diminuição de ruídos hidroaéreos, melena e dor à palpação em hipocôndrio e flanco direitos; seus controles hematimétricos, do $11^{\circ}$ ao $15^{\circ}$ dia de internação, apresentaram queda importante de hemoglobina de $12,5 \mathrm{~g} / \mathrm{dL}$ para 9,6 g/dL.

Diante do quadro, realizou-se tomografia computadoriza (TC) de abdome e pelve que indicou pneumoretroperitônio (Figura 1), mais evidente à direita, sugestivo de perfuração duodenal pós CPRE, sem identificação do ponto de perfuração no estudo.

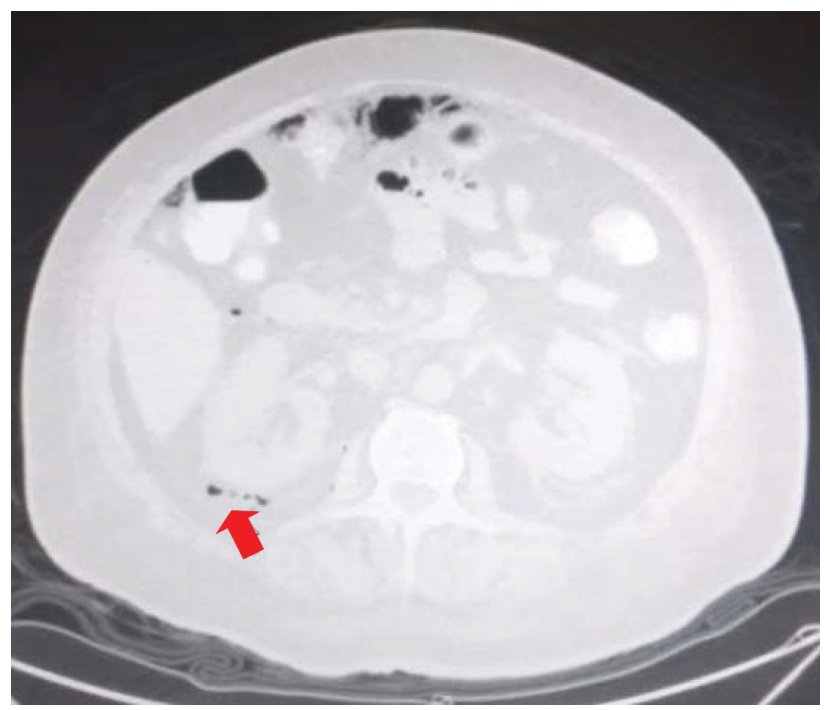

Figura 1 - Corte transversal de TC de abdome com evidência de ar livre em retroperitônio.

Após, à esofagogastroduodenoscopia (EDA), observou-se papila duodenal compatível com papilotomia com coágulo discreto em região distal e pequeno sangramento (Figura 2), tendo sido realizada hemostasia endoscópica terapêutica com injeção de adrenalina (Figura 3).

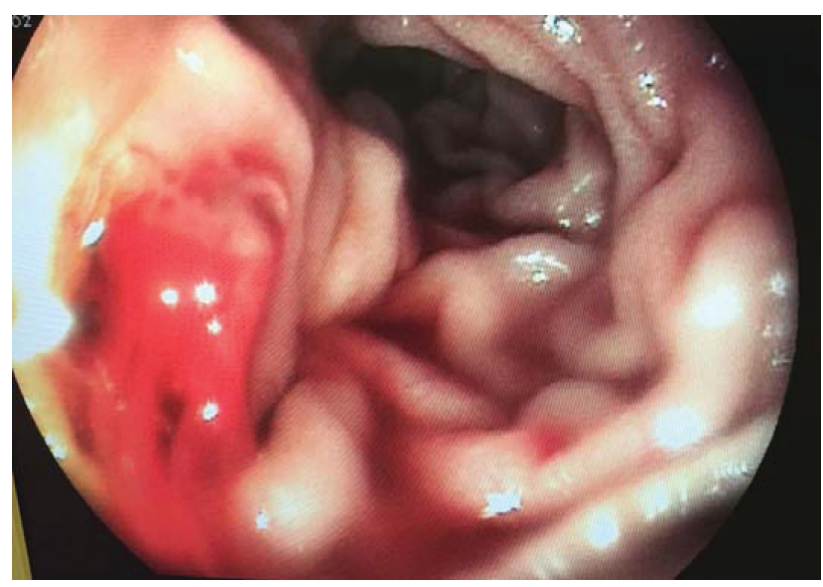

Figura 2 - Imagem de papila duodenal compatível com papilotomia com coágulo discreto e sangramento.

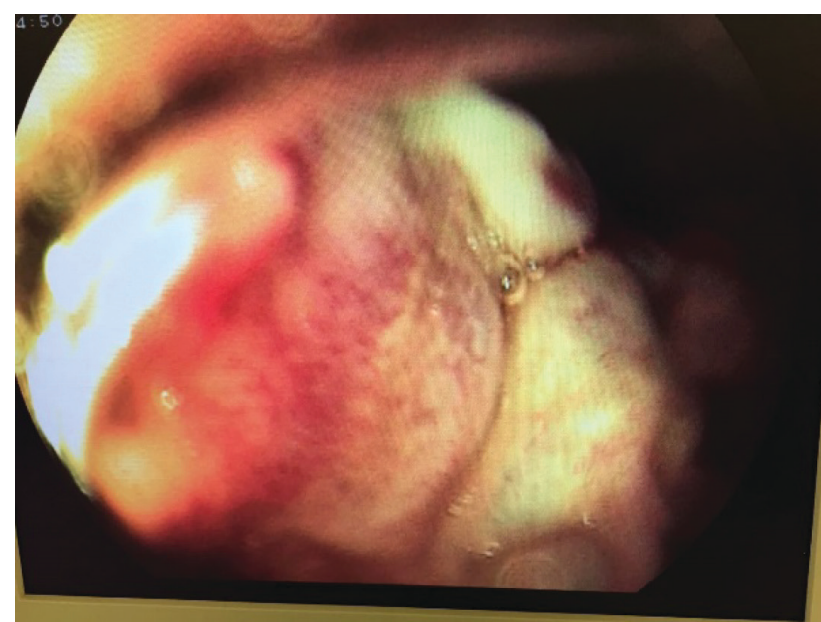

Figura 3 - Imagem de segmento duodenal após realização de hemostasia endoscópica terapêutica com injeção de adrenalina.

Após a EDA, houve persistência do quadro hemorrágico e queda da hemoglobina até $5,1 \mathrm{~g} / \mathrm{dL}$, sendo necessária transfusão sanguínea de 6 concentrados de hemácias e plasmas frescos congelados até que a paciente pode ser submetida à nova endoscopia, que evidenciou a manutenção do sangramento e presença de coágulos. Foi realizada injeção de solução de adrenalina, eletrocoagulação com plasma de argônio e clipagem endoscópica com 2 clipes, atingindo hemostasia efetiva.

Paciente evoluiu progressivamente bem com realização de colecistectomia posteriormente.

\section{Discussão}

A colangiopancreatografia retrógrada endoscópica, ou CPRE, é um método invasivo diagnóstico, 
Alves MMSR, Basile ML, Yamaoka PHT, Assef JC, De Campos T. Tratamento minimamente invasivo da coledocolitíase e suas complicações: relato de caso.

porém sobretudo terapêutico, que permite o acesso aos ductos hepáticos, pancreáticos e das vias biliares ${ }^{(3)}$.

Segundo a Sociedade Americana de Endoscopia Gastrointestinal, a indicação é restrita aos casos de: avaliação de desordens pancreáticas e biliares em caso de alterações objetivas em exames laboratoriais e de imagem; pancreatite aguda com colangite ou obstrução biliar; pancreatite crônica; paliação em casos de obstruções biliares malignas; tratamento padrão ouro em caso de vazamento biliar pós-operatório; e presença de cálculos ou interrupções nas vias analisadas ${ }^{(4-5)}$. Na prática, em casos de pacientes ictéricos com cálculo ao estudo ultrassonográfico, como o caso relatado, a CPRE está bem indicada ${ }^{(6)}$.

A efetividade diagnóstica e terapêutica da CPRE está relacionada ao sucesso da cateterização, que pode ser complicada. Na literatura, entretanto, as taxas de extração total dos cálculos por esse método chegam a $99 \%{ }^{(6)}$.

Dos procedimentos endoscópicos do trato gastrointestinal, embora segura, a CPRE está associada à maior taxa de complicações (2 a $20 \%$ ). Esse risco não é relacionado, estatisticamente, às características de gênero, idade e estado de saúde do paciente, mas sim à experiência do endoscopista que realiza o procedimento $^{(7)}$ e grau de dificuldade da realização da CPRE ${ }^{(1)}$.

Das possíveis complicações, as mais frequentes são pancreatite, hemorragia, colangite, alterações cardiopulmonares, infecções e perfuração duodenal ${ }^{(7-8)}$, sendo este último o mais raro, porém de grande gravidade, com mortalidade associada de 16 a $18 \%{ }^{\left({ }^{(8)}\right.}$. Pode decorrer da esfincterotomia (56\%), manipulação do fio guia $(23 \%)$, dilatação da estenose $(4 \%)$ e da inserção ou migração do stent $(3 \%)^{(9)}$.

O sangramento, por sua vez, corresponderia a uma porcentagem superior $(1,4 \%)^{(1)}$ das complicações, e estaria principalmente relacionado à pequenos centros de realização do procedimento ${ }^{(10)}$. Pode ser considerado leve, moderado ou grave de acordo com a necessidade de transfusão sanguínea ${ }^{(1)}$, de maneira que o caso do relato estaria enquadrado na terceira classificação.

Em casos de difícil cateterização, como o relato, em que o endoscopista encontrou resistência ao endoscópio devido a uma anomalia de implantação do colédoco, a esfinterotomia, ou papilotomia pré corte pode ser utilizada e aumentar a taxa de sucesso, embora agregue, na prática, um maior risco ao procedimento ${ }^{(11)}$. Em estudo retrospectivo com 14 pacientes que apresentaram perfuração duodenal pós CPRE do Centro Médico da Universidade da California do Sul, Stapfer et al, $2000^{(8)}$, concluíram que em $78 \%$ dos casos a perfuração pode ser constatada ou suspeitada já durante o exame. O laudo da CPRE já sugeria uma possível perfuração de maneira que os controles hematimétricos foram bem indicados.
Daher Filho et al (2007)(6) em análise retrospectiva de 178 casos do Grupos de Vías Biliares e Pâncreas da Faculdade de Ciências Médicas da Santa Casa de São Paulo, todavia, não encontraram associação estatística entre a papilotomia e a incidência de complicações pós CPRE e observaram a necessidade do procedimento em $78,7 \%$ dos casos analisados, sendo que somente $10,8 \%$ dos pacientes apresentaram eventos adversos, dos quais $0,6 \%$ apresentaram a perfuração propriamente dita e $2,2 \%$, sangramento.

Sabe-se, contudo, que a técnica de pré corte, a gastrectomia Billroth II, injeção de contraste em quantidade média, a duração do procedimento, disfunção do esfíncter de Oddi, ducto biliar comum dilatado e estenose biliar constituem fatores de risco para perfuração duodenal ${ }^{(9)}$. A técnica de pré corte em si estaria inclusive mais relacionada ao risco de hemorragias do que perfurações ${ }^{(12)}$. As coagulopatias, histórico de cirrose e a CPRE com fins terapêuticos estariam associados a maior risco de sangramento pós procedimento ${ }^{(13-14)}$.

No relato, paciente previamente estável evoluiu com náusea, taquicardia, distensão e dor abdominal, diminuição dos ruídos hidroaéreos e quedas hematimétricas pós CPRE, sugestivos de complicações. Solicitada TC, observou-se pneumoretroperitônio sugestivo de perfuração pequena o suficiente para inviabilizar a passagem de contraste, porém capaz de permitir passagem de ar e promover sangramento contínuo.

Em EDA, observou-se coágulo em região distal de papilotomia recente, com pequeno sangramento, tendo sido realizada hemostasia terapêutica efetiva em dois tempos.

Stapfer et $\mathrm{al}^{(8)}$ propuseram, em 2000, a classificação das perfurações quanto a sua localização anatômica em: (I) parede medial ou lateral do duodeno; (II) periampulares; (III) ducto biliar distal; e (IV) somente ar retroperitoneal sem perfuração verdadeira.

No caso, a paciente foi diagnosticada com uma perfuração da segunda porção duodenal por manipulação endoscópica com esfincterotomia, configurando uma lesão tipo II na classificação de Stapfer e a Síndrome da Janela Posterior.

Embora não exista ainda consenso acerca do manejo da perfuração duodenal pós CPRE, observa-se que a tendência inicial de tratamento cirúrgico tem progressivamente cedido espaço para a abordagem conservadora com antibioticoterapia e observação, ao menos nos casos em que não há quadro de sepse, peritonite, coleção de fluidos e nem grandes perfurações $^{(8,9,15)}$.

As lesões do tipo II tendem a cura espontânea e geralmente não necessitam de abordagem cirúrgica ${ }^{(8)}$, se valendo de cuidados conservadores, como sonda nasogástrica, antibioticoterapia, suporte clínico, ou tratamento endoscópico. 
O sangramento pós CPRE, por sua vez, apresenta diversas modalidades de correção endoscópica ${ }^{(10)}$; no caso de hemorragia grave de difícil controle, como o relatado, há necessidade de associação de métodos, além da transfusão sanguínea.

A principal estratégia para a redução dos riscos desse procedimento é a sua indicação inequívoca e a realização por endoscopistas com larga experiência ${ }^{(7)}$.

\section{Conclusão}

Embora seja um método terapêutico seguro, a CPRE, apresenta riscos aos pacientes a ela submetidos e a prevenção dos eventos adversos se baseia na correta indicação do procedimento. As dificuldades técnicas no momento do procedimento estão relacionadas a um maior índice de complicações.

\section{Referências Bibliográficas}

1. Coelho-Prabhu N, Shah ND, Van Houten H, Kamath PS, Baron TH. Endoscopic retrograde cholangiopancreatography: utilisation and outcomes in a 10-year population-based cohort. BMJ Open .2013; 3(5): pii: e002689.

2. Schiavon LL, Rodrigues RA, Nakao FS, Di Sena VO, Ferrari AP, Libera ED Jr. Subcutaneous emphysema, pneumothorax and pneumomediastinum following endoscopic sphincterotomy. Gastroenterology Res. 2010; 3(5):216-8.

3. Ahmed M, Kanotra R, Savani GT, Kotadiya F, Patel N, Tareen $\mathrm{S}$, et al. Utilization trends in inpatient endoscopic retrograde cholangiopancreatography (ERCP): A cross-sectional US experience. Endosc Int Open. 2017; 5(4):E261-71.

4. Adler DG, BaronTH, Davila RE, Egan J, Hirota WK, Leighton JA, et al. ASGE guideline: the role of ERCP in diseases of the biliary tract and the pancreas. Gastrointest Endosc, 2005; 62(1):1-8.

5. Chathadi KV, Chandrasekhara V, Acosta RD, Decker A, Early DS, Eloubeidi MA, et al. The role of ERCP in benign diseases of the biliary tract. Gastrointest Endosc. 2015;81(4):795-803.

6. Daher Filho PF, De Campos T, Kuryura L, Belotto M, Silva RA, Pacheco Jr AM. Avaliação de complicações relacionadas à CPRE em pacientes com suspeita de coledocolitíase. Rev Col Bras Cir. 2007; 34(2):114-8.

7. Freeman ML. What are the complications (adverse effects) of ERCP? In: NIH State-of-the-Science Conference on Endoscopic Retrograde Cholangiopancreatography (ERCP) for Diagnosis and Therapy. William H. Natcher Conference Center. National Institutes of Health. Bethesda, Maryland. Bethesda (Maryland): NIH; 2002. p. 91-2.

8. Stapfer M, Selby RR, Stain SC, Katkhouda N, Parekh D, Jabbour $\mathrm{N}$, et al. Management of duodenal perforation after endoscopic retrograde cholangiopancreatography and sphincterotomy. Ann Surg.2000; 232(2):191-8.

9. Paspatis GA, Dumonceau JM, Barthet M, Meisner S, Repici A, Saunders BP, et al. Diagnosis and management of iatrogenic endoscopic perforations: European Society of Gastrointestinal Endoscopy position statement. Endoscopy. 2014;46(8):693-711.

10. Loperfido S, Angelini G, Benedetti G, Chivoli F, Costan F, De Bernardinis F, et al. Major early complications from diagnostic and therapeutic ERCP: a prospective multicenter study. Gastrointest Endosc. 1998; 48(1):1-10.

11. Lehman GA. What Are the Determinants of Success in Utilization of ERCP in the Setting of Pancreatic and Biliary Diseases? In: National Institutes of Health. NIH State-of-the-Science Conference on Endoscopic Retrograde Cholangiopancreatography (ERCP) for Diagnosis and Therapy. William H. Natcher Conference Center. Bethesda (Maryland): NIH; 2002. p. 93-6.

12. Donatelli G, Dumont JL, Cereatti F, Tuszynski T, Vergeau BM, Meduri B. Revision of biliary sphincterotomy by re-cut, ballon dilatation or temporary stenting: comparison of clinical outcome and complication rate (with vídeo). Endosc Int Open. 2017; 5(3):E395-401.

13. Abdulsamad M, Reddy P, Guvvala S, Dev A. Recombinant fator VIIa use for Endoscopic Retrograde Cholangiopancreatography and unusual coagulopathy. Gastroenterology Res. 2017; 10(2):144-6.

14. Novaneethan V, Njei B, Zhu X. Safety of ERCP in patients with liver cirrhosis: a national database study. Endosc Int Open. 2017; 5(4): E303-14.

15. Kumbari V, Sinha A, Reddy A, Afghani E, Cotsalas D, Patel YA, et al. Algorithm for the management of ERCP-related perforations. Gastrointest Endosc. 2016; 83(5):934-43.

Artigo recebido: 05/06/2017

Artigo aprovado: 16/11/2017 